Pract. oto-rhino-laryng. 1965;27:323

\title{
An Uncommon Case of Herpes Zoster Oticum
}

W.R.

Kneib

\section{Rotterdam}

Author's address: W. R. Kneib M.D., E.N.T. Clinic, Dijkzigt Ziekenhuis, Rotterdam (Netherlands)

A description is given of the symptomatology of herpes zoster oticum in a fifty-nine-year-old female.

On account of this and in view of data from literature an affection of the ganglion geniculi is considered unlikely. Poly-radiculo-encephalitis was diagnosed. 